\title{
A comparative investigation of the effect of ondansetron and dexamethasone in tonsillectomy of children aged 6-10 in Shahrood hospitals
}

\author{
Negar Hamidi* \\ Iran University of Science and Technology, Iran
}

\section{Introduction and purpose}

Tonsillectomy is one of the most common surgeries among children throughout the world. One of the common post-operative complications is nausea and vomiting, about $70 \%$ of which occur within the first 24 hours after surgery.

It can lead to aspiration of contents of stomach, water and electrolyte disorders, hypovolemia, delayed discharge, rehospitalization, higher hospital costs and lower patient satisfaction [1]. It also seems that tonsillectomy stimulates the labyrinth, and subsequently chemoreceptor trigger zone (CTZ) which is located next to it and exacerbates nausea and vomiting. Therefore, its control is of great importance [2]. The incidence of this complication is related to several factors such as the type of surgery, the technique and the type of drugs used in anaesthesia, as well as post-operative factors such as pain, movement and the use of narcotics. Drugs such as antihistamines, butyrophenones, dopamine and serotonin and benzodiazepine antagonists, etc. are used to prevent and treat nausea and vomiting, each of which has its own benefits and side effects such as excessive sedation, dry mouth, hypotension, dysphoria, hallucinations, and extrapyramidal symptoms. Additionally, the high price of some of these drugs such as serotonin antagonists has made the use of these drugs difficult. Steroids, especially dexamethasone, can reduce nausea and vomiting by directly affecting the respiratory centre as well as inhibition of chemical mediators. In addition, drugs such as anticholinergics and anti-serotonins are effective in controlling this disorder [3]. This annoying problem (nausea and vomiting) can be reduced by replacing intravenous anaesthesia with inhalational anaesthesia and taking medications. However, despite the use of new anaesthetics and antiemetic drugs, the incidence of this complication remains high.

Several studies have been conducted to compare different antiemetics, but no successful treatment has been found. Since many studies that compared the two drug groups produced different results, the researcher sought to accurately compare the effect of ondansetrondexamethasone on tonsillectomy postoperative nausea and vomiting among children aged 6-10 years in Shahrood hospitals.

\section{Methodology}

This study was a randomized double-blind clinical trial. The population of the study included all children aged 6-10 years undergoing tonsillectomy surgery in Shahrood hospitals. Criteria for entering the study included 6-10 years of age, lack of previous history of using sedatives and antiemetics, lack of allergy to ondansetron and dexamethasone, no drug use and knowledge, consent and awareness on the methodology of the study. Then, 80 eligible children were randomly divided into two groups of 40 . Forty patients were assigned to a group receiving ondansetron injections and 40 were assigned to the group receiving dexamethasone injections. The first group received $0.1 \mathrm{mg} / \mathrm{kg}$ of ondansetron IV injection and the second group received 0.5 to maximum $8 \mathrm{mg} / \mathrm{kg}$ of dexamethasone. After the anaesthesia and intubation of the patient and before the tonsillectomy operation, the mentioned drugs were prescribed to both groups of patients. At the end of the operation, patients were transferred into the recovery room after removing the contents and secretions of their stomach and mouth using sonda nelaton and neutralizing the muscle block by the atropine and neostigmine. Then, an anaesthesiologist who had no information about the injection and the project followed up and recorded the presence or absence, and the number and frequency of postoperative nausea and vomiting sequentially at 1-6 and 6-12 hours. The obtained data were analyzed using t-test and chi-square with SPSS version 21 and $P<0.05$ statistical significance level was used.

\section{Results}

The results showed that $29(72.50 \%)$ participants were male and 11 (27.5\%) participants were female. In dexamethasone group, 26 (65\%) participants were male and $14(35 \%)$ participants were female. The frequency of postoperative nausea and vomiting in recovery room in ondansetron group was $57.5 \%$ and $47.5 \%$ respectively; this frequency was $40 \%$ and $25 \%$ in the dexamethasone group respectively. In the sixth hour after the operation, the frequency of nausea and vomiting was $45 \%$ and $30 \%$ respectively in the ondansetron group; this frequency was $25 \%$ and $17.5 \%$ in the dexamethasone group. In addition, in the $12^{\text {th }}$ hour after the operation, the frequency of nausea and vomiting was $30 \%$ and $22.5 \%$ respectively in the ondansetron group while in the dexamethasone group, the frequency of nausea and vomiting was $0 \%$. The results of this study indicated that dexamethasone and ondansetron yielded the lowest frequency in patient nausea and vomiting respectively.

The results also showed that there was a significant difference between the prescription of ondansetron and dexamethasone in preventing the incidence of tonsillectomy post-operative nausea

${ }^{\star}$ Correspondence to: Negar Hamidi, Iran University of Science and Technology, Iran, E-mail: negar_hamidi@hotmail.com

Received: December 12, 2018; Accepted: December 24, 2018; Published: December 28, 2018 
and vomiting. Dexamethasone had a more effective role in reducing postoperative vomiting 6-12 hours after the operation compared to ondansetron $(P=0.039)$. However, there is no significant difference between the two drugs in the recovery room after the operation with regard to their effect on reducing the frequency of nausea and vomiting $(P=0.41)$.

\section{Discussion}

The results of this study showed that dexamethasone is more effective than ondansetron in reducing the incidence of tonsillectomy post-operative nausea and vomiting and maximum effect is observed in the first 6 hours after the operation. Steroids, especially dexamethasone, can be effective in reducing the frequency of nausea and vomiting with direct effect on the respiratory center and inhibition of chemical mediators.

\section{Conclusion}

Therefore, according to the results of this study, intravenous dexamethasone preoperative injection in children can reduce nausea and vomiting complications and subsequently reduce the cost of treatment and duration of hospitalization. Therefore, it is suggested to use dexamethasone to prevent tonsillectomy post-operative nausea and vomiting.

\section{References}

1. Bolton CM, Myles PS, Nolan T, Stern JA (2006) Prophylaxis of post-operative vomiting in children undergoing tonsillectomy. Br J Anaesth 97: 593-604. [Crossref]

2. Donlon JV (2005) Anesthesia for Eye, Ear, Nose, and throat surgery. In Miller RD. Textbook of miller's anesthesia. 6th Ed. New York: Natasha Andjelkavic. 2543-2544.

3. Ghiamat MM (2003) The important complications in pre and post-surgery. Tabib J 7: 122-125.

Copyright: $@ 2018$ Hamidi N. This is an open-access article distributed under the terms of the Creative Commons Attribution License, which permits unrestricted use, distribution, and reproduction in any medium, provided the original author and source are credited. 